\title{
Correction
}

\section{On Realization of Nonlinear Systems Described by Higher-Order Differential Equations*}

\section{A. J. van der Schaft}

Department of Applied Mathematics, Twente University of Technology, P.O. Box 217, 7500 AE Enschede, The Netherlands

In Section 6 of the above paper it was shown how under constant rank assumptions an external system

$$
R_{i}\left(w, \dot{w}, \ldots, w^{(k)}\right)=0, \quad i=1, \ldots, l, \quad w \in \mathbb{R}^{q},
$$

can be transformed to an equivalent external system, satisfying either the main assumption (Assumption 4), and so admits a driven realization, or is inconsistent. The class of local equivalence transformations (see (6.21)) which had to be used for this purpose was however not correctly stated. In fact this class has to be enlarged in the following way. Suppose as in (6.28) that

$$
A_{j}=\lambda_{1} A_{1}+\cdots+\lambda_{j-1} A_{j-1}+\lambda_{j+1} A_{j+1}+\cdots+\lambda_{l} A_{l}
$$

where (see (6.30)-(6.31))

$$
A_{i}=\left(\frac{\partial}{\partial w_{1}^{\left(k-\rho_{j}\right)}} L_{g_{0}}^{\left(\rho_{i}-\rho_{j}\right)} R_{i}, \ldots, \frac{\partial}{\partial w_{q}^{\left(k-\rho_{j}\right)}} L_{g_{0}}^{\left(\rho_{i}-\rho_{j}\right)} R_{i}\right)
$$

with $\rho_{i} \geq \rho_{j}, i=1, \ldots, l$. By definition of the integers $\rho_{i}$ the function $R_{j}=L_{g_{0}}^{0} R_{j}$ and the $l-1$ functions $L_{g_{0}}^{\left(\rho_{i}-\rho_{j}\right)} R_{i}, i=1, \ldots, j-1, j+1, \ldots, l$ only depend on $w^{\left(k-\rho_{j}\right)}:=\left(w_{1}^{\left(k-\rho_{j}\right)}, \ldots, w_{q}^{\left(k-\rho_{j}\right)}\right)$ and on $\left(w_{1}^{(k-r)}, \ldots, w_{q}^{(k-r)}\right)$ for $r=\rho_{j}+1, \ldots, k$. Denote these last remaining coordinates by $z$. Now apply Lemma 6.2 to $R_{j}$ and $L_{g_{0}}^{\left(\rho_{i}-\rho_{j}\right)} R_{i}, i=1, \ldots, j-1, j+1, \ldots, l$, seen as functions of $w^{\left(k-\rho_{j}\right)}$, depending on the parameters $z$. This yields for every $z$ the existence of a smooth function 
$\varphi_{z}: U \subset \mathbb{R}^{l} \rightarrow \mathbb{R}$, with $U$ an open neighborhood of $0 \in \mathbb{R}^{\prime}$, such that (see (6.33)(6.34))

$$
\begin{gathered}
\varphi_{z}(0, \ldots, 0)=0, \quad \frac{\partial \varphi_{z}}{\partial y_{1}}(0,0, \ldots, 0) \neq 0 \\
\frac{\partial}{\partial w_{s}^{\left(k-\rho_{j}\right)}} \varphi_{z}\left(R_{j}, L_{g_{0}}^{\left(\rho_{1}-\rho_{j}\right)} R_{1}, \ldots, L_{g_{0}}^{\left(\rho_{j-1}-\rho_{j}\right)} R_{j-1},\right. \\
\left.L_{g_{0}}^{\left(\rho_{j+1}-\rho_{j}\right)} R_{j+1}, \ldots, L_{g_{0}}^{\left(\rho_{l}-\rho_{j}\right)} R_{l}\right)=0
\end{gathered}
$$

for $s=1, \ldots, q$. Now define

$$
\psi\left(y_{1}, \ldots, y_{l}, z\right):=\varphi_{z}\left(y_{1}, \ldots, y_{l}\right) .
$$

(Notice that $\varphi_{z}$ can be chosen in such a way that $\psi$ is also smooth.) Then $\psi$ defines a local equivalence transformation of system (1) to an equivalent external system by replacing the $j$ th equation $R_{j}\left(w, \dot{w}, \ldots, w^{(k)}\right)=0$ in (1) by

$$
\psi\left(R_{j}, L_{\mathbf{g}_{0}}^{\left(\rho_{1}-\rho_{j}\right)} R_{1}, \ldots, L_{g_{0}}^{\left(\rho_{j-1}-\rho_{j}\right)} R_{j-1}, L_{g_{0}}^{\left(\rho_{j+1}-\rho_{j}\right)} R_{j+1}, \ldots, L_{g_{0}}^{\left(\rho_{1}-\rho_{j}\right)} R_{l}, z\right)=0 .
$$

(Compare with (6.35); the difference lies in the dependence of $\psi$ on the $z$ coordinates.) Now everything works the same way as in the above paper. For the newly defined external system the numbers $\rho_{i}$ are the same, except for the $j$ th one which is by (5) strictly larger than $\rho_{j}$. If the $A$-matrix of this newly defined system equals $l$ then we are done. Otherwise, we can repeat the above procedure, and the conclusion (Theorem (6.3)) follows. 